\section{Einfach wegwaschen}

Wenn Pollen fliegen, verfangen sie sich leicht und in erstaunlichen Mengen in den Haaren und gelangen auf diesem Weg auch ins Kopfkissen. Um die Belastung nachts zu reduzieren ist es bei Pollenallergie sinnvoll, die Haare täglich vor dem Zubettgehen mit einem milden Shampoo zu Waschen. Das wird jetzt in der Apotheke speziell für Heuschnupfenpatienten beworben. Die dermatologische Haarpflege Ducray bietet mit Elution ein Shampoo für die häufige Anwendung bei sensibler und gereizter Kopfhaut. Wirkstoffe wie Zinkglukonat und Glycin beruhigen die Kopfhaut, schützen sie vor äußeren Angriffen und mildern Hautirritationen.

Nach Informationen von Pierre Fabre Dermo-Kosmetik, Freiburg

\section{Bequem gepflastert}

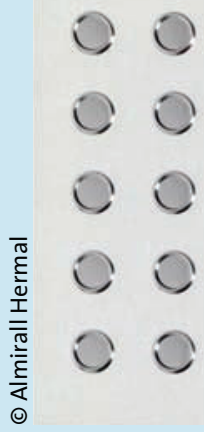

Ein neues patienten- und anwenderfreundliches Epikutan-Testpflaster bietet das deutsche Unternehmen Almirall Hermal ab sofort an. Pro Pflaster sind zehn Aluminiumkammern auf ein Vlies aus Rayon-Fasern aufgebracht. Wie von der Deutschen Kontaktallergie-Gruppe empfohlen, gewährleistet das Pflaster so Okklusion bei der Testung. Als Klebstoff wurde ein für medizinische Zwecke entwickelter Acrylatkleber verwendet. Der Kleber ist frei von Kolophonium und hypoallergen. Durch eine Perforation lässt sich der beschriftete Pflasterabschnitt leicht vom Pflaster abtrennen. Ebenfalls bequem von einem Bogen zu entnehmen sind die 100 Filterpapierscheiben, die in jeder Packung enthalten sind. Weitere Details können per E-Mail aninfo@almirall.de oder telefonisch unter der Nummer (o 40) 727 04-2 29 erfragt werden.

Nach Informationen von Almirall, Reinbek

\section{0 \\ Gute Asthmakontrolle: Wunsch oder Wirklichkeit?}

W ie Asthmakranke ihre Erkrankung wahrnehmen, mit ihr umgehen und sie anderen gegenüber beschreiben, sollte die im Jahr 2010 durchgeführte Studie Asthma Insight and Management in Europe and Canada (EUCAN AIM) zeigen. Dazu befragte ein US-Meinungsforschungsinstitut in Zusammenarbeit mit der European Federation of Asthma and Allergy Associations (EFA) 2.420 Asthmapatienten aus Kanada ( $\mathrm{n}=401)$ und fünf europäischen Ländern. Neben Deutschland (n = 402) waren dies Frankreich $(\mathrm{n}=417)$, Italien, Spanien und Großbritannien (jeweils n = 400). Die Ergebnisse zeigten, dass eine gute Asthmakontrolle gemäß den aktuellen Behandlungsleitlinien der Global Initiative for Asthma (GINA) nach wie vor für viele Asthmapatienten ein unerreichtes Ziel ist.

Unter den in Deutschland befragten Patienten schätzten vier von fünf $(82 \%)$ ihre Asthmaerkrankung als vollständig unter Kontrolle oder gut kontrolliert ein. Mit drei bis vier akuten Asthmaschüben oder nur zwei notfallmäßigen Arztbesuchen aufgrund ihres Asthmas im Jahr ordneten dabei mehr als die Hälfte der Patienten ihre Asthmaerkrankung als gut kontrolliert ein (52 bzw. 64\%).

Offensichtlich gibt es aber eine Diskrepanz zwischen empfundenem und tatsächlichem Grad der Asthmakontrolle. Im vergangenen Jahr erlitt nämlich mehr als die Hälfte (52\%) der befragten Patienten mindestens einen schweren Asthmaanfall. Knapp 18\% der Patienten wurden aufgrund akuter Asthmasymptome in ein Krankenhaus eingewiesen, mussten die Notaufnahme eines Krankenhauses aufsuchen oder anderweitig medizinisch notversorgt werden. Im Verlauf der Erkrankung hatte sogar über ein Drittel (39\%) schon Todesangst aufgrund eines akuten Asthmaanfalls, $14 \%$ davon im letzten Jahr. Da- rüber hinaus musste auch annähernd ein Drittel (35\%) der befragten Asthmapatienten mindestens einmal in der Woche ein Bedarfsmedikament als Reliever inhalieren, um Asthmasymptome zu lindern. Ein Großteil der Patienten gab an, dass bei ihnen schwere Asthmaanfälle in Ruhe (27\%), im Schlaf (30\%) oder während des Sprechens (25\%) aufgetreten waren. Mehr als die Hälfte (57\%) verzichtete aufgrund schwerer Atemnotanfälle gänzlich auf Sport. Von krankheitsbedingten Fehlzeiten in der Schule oder am Arbeitsplatz im Laufe ihrer Asthmaerkrankung berichtete ein Drittel (32\%) der Befragten.

Wunsch und Wirklichkeit klaffen demnach auseinander: Schwere Asthmaepisoden schränken Lebensqualität und Alltagsaktivitäten erheblich ein. Das muss nicht so bleiben. „Mit dem richtigen Asthmamanagement gemäß der aktuellen Behandlungsleitlinien können Asthmapatienten langfristig schweren Asthmaepisoden vorbeugen und ein aktives, produktives Leben führen“, riet Marianella Salapatas, die Präsidentin der EFA. red

Nach Informationen von MSD, Haar 